\title{
Isokinetic Assessment of the Wrist Muscles in Females With Fibromyalgia
}

\author{
Hayal GÜLER, Mustafa Turgut YILDIZGÖREN, Nilgun ÜSTÜN, Hacer PAKSOY, Ayşe Dicle TURHANOĞLU \\ Department of Physical and Rehabilitation, Medical Faculty of Mustafa Kemal University, Hatay, Turkey
}

\begin{abstract}
Objectives: This study aims to evaluate wrist muscle strength and muscle fatigue in females with fibromyalgia syndrome (FMS) and compare the results with those of healthy controls.

Patients and methods: Thirty consecutive female FMS patients (mean age 39.8 \pm 6.7 years; range 25 to 49 years) and 50 age and body mass index similar healthy females (mean age $35.4 \pm 7.9$ years; range 27 to 48 years) were enrolled. Patients' clinical characteristics were recorded and symptoms were evaluated by the Fibromyalgia Impact Questionnaire. In addition to the demographic characteristics, physical activities of all subjects were questioned, isokinetic muscle performance was measured, and fatigue index was calculated by endurance test.

Results: The peak torque values of the wrist extensor and flexor muscles (at an angular velocity of $90 \%$ second) were higher in the control group than in the FMS group (both $\mathrm{p}<0.01$ ). There were no differences between the groups in terms of the fatigue indexes of the flexor and extensor muscles of the wrist (both $p>0.05$ ). While there were differences between the groups regarding weekly hours of walking ( $p=0.01)$ and house cleaning ( $p<0.001)$, no differences were determined for weekly hours of bicycling, gardening, doing sports, or total physical activity. There was no correlation between the peak torque values and clinical characteristics in FMS group.

Conclusion: Patients with FMS had decreased muscle strength compared to healthy controls. Further studies with larger participants are needed to explain the relationship between upper limb muscle performance and FMS, as well as the underlying pathogenesis.

Keywords: Dynamometer; fatigue index; fibromyalgia; wrist muscle.
\end{abstract}

Fibromyalgia syndrome (FMS) is defined as a chronic, nonarticular rheumatic disorder characterized by generalized muscle pain, stiffness, fatigue and disturbed sleep. FMS is six times more common in females than males and mostly develops in females between 20 and 55 years of age. Prevalence of FMS in general population changes between $2 \%$ to $8 \% .{ }^{1}$ Although various mechanisms have been suggested, the underlying pathophysiological mechanism is currently unknown. ${ }^{1,2}$ In addition to the researches focusing on genetic basis of FMS, several attempts have been made to identify abnormalities in the muscle tissue of FMS patients because of the fact that muscle pain is the main symptom. ${ }^{3}$ Bengtsson $^{4}$ indicated decreases in adenosine triphosphate and phosphocreatine levels and the appearance of ragged red fibers in the tender points of the trapezius muscle of FMS patients. This might reflect the sequelae of continuous muscle microtrauma leading to increased post-exertional pain and other painful symptoms in FMS patients.

Prolonged muscle tension and ischemia have been suggested to cause painful symptoms in FMS, ${ }^{5}$ which in turn lead to decreased muscle performance. In many FMS studies, knee extensor and flexor muscle performance have been evaluated and compared with control groups. ${ }^{6-11}$ Contrary to lower limb, there are fewer studies 
evaluating upper limb muscle performance in FMS patients. In addition, widespread pain, weakness and fatigue are common complaints in the upper limbs of the FMS patients. Accordingly, in this study, we aimed to evaluate wrist muscle strength and muscle fatigue in females with FMS and compare the results with those of healthy controls.

\section{PATIENTS AND METHODS}

Thirty consecutive female patients (mean age $39.8 \pm 6.7$ years; range 25 to 49 years) who were admitted to our outpatient clinic with a diagnosis of FMS ${ }^{12}$ between July 2014 and July 2015 at the Mustafa Kemal University Medical School, Department of Physical Medicine and Rehabilitation, and 50 age and body mass index similar female healthy subjects (mean age $35.4 \pm 7.9$ years; range 27 to 48 years) were enrolled. All patients and controls were housewives. Subjects with concomitant diagnoses affecting muscle strength measurements such as arthritis, osteoarthritis, previous history of muscle trauma or any orthopedic procedures, and cardiovascular, endocrinologic, neurologic, and psychiatric problems, those using medications such as statins, corticosteroid which are likely to influence muscle function, and those having risks for muscle performance tests were excluded. Clinical assessment was performed by a blinded physician with a detailed, standardized form. Demographic and clinical features of the patients including age, body mass index, disease duration, and tender point count (digital palpation technique) were recorded. The study protocol was approved by the local ethics committee. A written informed consent was obtained from each subject. The study was conducted in accordance with the principles of the Declaration of Helsinki.

The subjects were asked about how much time (average hours per week) they spent on activities including walking, bicycling, gardening, housekeeping, and sports. ${ }^{13}$ Thus, total physical activity of each subject was measured.

The Turkish version of the Fibromyalgia Impact Questionnaire was used to assess FMS-related symptoms. ${ }^{14}$ Fibromyalgia Impact Questionnaire comprises 10 subscales including physical impairment, overall well-being, work missed, work difficulty, pain, fatigue, morning tiredness, stiffness, anxiety, and depression. The last seven subscales are scored using the visual analog scale with $1 \mathrm{~cm}$ increments. The total score ranges from 0 to 100 ; the higher score indicates a greater impact of the FMS.

Isokinetic muscle performance was measured using a calibrated isokinetic test device named Humac $^{\circledR} /$ NormTM Testing \& Rehabilitation System (Computer Sports Medicine Inc., Stoughton, MA, USA). The measurements were performed according to the procedure described previously. ${ }^{15}$ First, the subjects were instructed to sit on the testing chair with the forearm supported and in full pronation and the elbow flexed at about $90^{\circ}$. The joint axis of the wrist was aligned with the rotational axis of the dynamometer. Range of motion was $90^{\circ}\left(30^{\circ}\right.$ in dorsal flexion and $60^{\circ}$ in palmar flexion from the neutral position of the wrist). Weight of the hand was measured and eliminated at the beginning of the experiment for gravity correction. Concentric isokinetic contractions were measured first for dorsal flexion and then for palmar flexion. After a warm-up of five submaximal repetitions of wrist extension and flexion, concentric tests were applied at speeds of $90 \%$ second first, followed by $180 \%$ second. The isokinetic test protocol was as follows; five-repetitive at $90 \%$ second, 60 seconds resting period, and 15 -repetitive at $180 \%$ second. Tests were performed randomly to avoid bias. The measurements were carried out in the dominant side for all subjects. Measurements of torque, velocity, and position were performed over the entire range of motion. Their corresponding output signals were sampled simultaneously and digitalized for analysis through an external device designed for the present study. ${ }^{16}$ The fatigue index is the declined percentage (\%) peak torque during the endurance test and calculated as the percentage change from the initial and final peak torques.

\section{Statistical analysis}

Data were analyzed using the SPSS version 16.0 (SPSS, Inc., Chicago, IL, USA) program. Data were expressed as median (minimum-maximum). Mann-Whitney $U$ test was used to compare differences between the groups. The correlation between clinical variables was analyzed with the 
Table 1. Demographic and clinical characteristics of study subjects

\begin{tabular}{|c|c|c|c|c|c|}
\hline & \multicolumn{2}{|c|}{ Fibromyalgia syndrome group $(\mathrm{n}=30)$} & \multicolumn{2}{|c|}{ Control group $(n=50)$} & \multirow[b]{2}{*}{$p$} \\
\hline & Median & Min.-Max. & Median & Min.-Max. & \\
\hline Age (years) & 38 & $25-49$ & 35 & $27-48$ & 0.066 \\
\hline Body mass index $\left(\mathrm{kg} / \mathrm{m}^{2}\right)$ & 26.3 & $21.6-40.1$ & 25.45 & $19.1-40.4$ & 0.233 \\
\hline Disease duration (years) & 3 & $1-15$ & - & - & \\
\hline Number of tender points & 14 & $11-18$ & - & - & \\
\hline Visual analog scale (mm) & 70 & $30-90$ & - & - & \\
\hline Fibromyalgia Impact Questionnaire scores & 58.1 & $29.6-80$ & - & - & \\
\hline \multicolumn{6}{|l|}{ Physical activity (h/wk) } \\
\hline Walking & 0.5 & $0-15$ & 3.5 & $0-14$ & 0.011 \\
\hline Bicycling & 0 & $0-2$ & 0 & $0-7$ & 0.302 \\
\hline Gardening & 0 & $0-30$ & 0 & $0-20$ & 0.081 \\
\hline Sports & 0 & $0-7$ & 0 & $0-14$ & 0.065 \\
\hline House cleaning & 14 & $0-35$ & 4 & $0-35$ & $<0.001$ \\
\hline Total & 15.5 & $1-60$ & 12.5 & $2-50$ & 0.116 \\
\hline \multicolumn{6}{|l|}{ Peak torque values at $90 \%$ s $(\mathrm{Nm})$} \\
\hline Wrist extensors & 5 & $3-11$ & 8 & 4-14 & $<0.001$ \\
\hline Wrist flexors & 8 & $4-14$ & 9 & $4-16$ & 0.005 \\
\hline \multicolumn{6}{|l|}{ Fatigue values at $180 \%$ s (\%) } \\
\hline Wrist extensors & 14 & $2-45$ & 14 & $0-84$ & 0.626 \\
\hline Wrist flexors & 16 & $0-82$ & 13 & $0-85$ & 0.392 \\
\hline
\end{tabular}

Spearman rank test. Statistical significance was set at $\mathrm{p}<0.05$.

\section{RESULTS}

The demographic and clinical characteristics of study subjects are shown in Table 1 . The peak torque values of the wrist extensor and flexor muscles at an angular velocity of $90 \%$ second were found to be lower in the FMS group. Regarding weekly hours of physical activity, while there were differences between the groups in walking $(p=0.011)$ and house cleaning $(p<0.001)$, no differences were determined in bicycling, gardening, doing sports, and total physical activity. All patients (100\%) had fatigue, $26(86.7 \%)$ had headache, 16 (53.3\%) had paresthesia, 13 (43.3\%) had irritable bowel syndrome, 24 (80\%) had sleep disorder, and three (10\%) had Raynaud's phenomenon. There was no correlation between the peak torque values and clinical characteristics in FMS group.

\section{DISCUSSION}

In this study, we attempted to observe whether wrist muscle strength and muscle fatigue resistance values of the FMS patients were any different from that of normal healthy subjects. According to our results, wrist flexor/extensor muscle strength values of FMS patients were lower than those of the healthy controls.

Several studies have evaluated muscle strength in FMS patients, most of which have indicated reproducible and significant reductions in the strength and performance of FMS patients as compared to healthy subjects. The percentage of reduction has been reported to be between 30\% to $40 \%$ in many studies and has changed based on the muscle groups and the type of exercise..$^{7-11,17,18}$

Measurement of muscle strength is generally performed on the knee extensors and flexors and only a few studies have examined muscle strength of upper extremity muscle groups. It has been shown that FMS patients had decreased knee muscle strength. ${ }^{9,10,19}$ Borman et al. ${ }^{11}$ found lower maximal voluntary muscle strength of the quadriceps and submaximal aerobic performance scores in the FMS patients, which were attributed to the impaired muscle function in these patients. Additionally, they reported that the decreased muscle performance in FMS patients was not related with the clinical findings, including pain severity, number of tender points, and duration of the symptoms. Yetişgin et al. ${ }^{20}$ compared FMS patients and controls with respect to proximal or distal muscle strength and muscle 
fatigue resistance values, and reported lower isokinetic values in FMS patients in hip and ankle muscle measurements. They also reported that the isokinetic muscle strengths were not associated with the disease-related parameters in FMS patients. ${ }^{20}$ To the best of our knowledge, we have studied wrist muscles of the upper extremity of FMS patients and shown that these patients had reduced isokinetic muscle strengths when compared with healthy subjects for the first time in the literature.

Only a few studies have investigated muscle strength of upper extremity muscle groups. ${ }^{17,18,21,22}$ Mengshoel et al. ${ }^{17}$ evaluated 26 females with FMS for muscle strength, aerobic capacity, and fatigue and measured grip strength using a manometer. The patients had lower grip strength, performed lesser repetitions with maximal grip pressure (dynamic endurance work), and kept their upper extremity in a fixed position for a lesser duration (static endurance work) as compared to the healthy matched controls. Köklü et al. ${ }^{23}$ reported that FMS patients had decreased handgrip strength as compared to the controls. Contrary to these studies, Akyol et al. ${ }^{19}$ did not find any difference between the FMS patients and healthy controls regarding handgrip strength. In the present study, we found the wrist extensor and flexor muscle strengths to be lower in the FMS patients. Reduced muscle performance and fatigue resistance caused by widespread pain may have negative impact on daily life. Further research is needed to identify causality of decreased muscle performance.

Increased muscle fatigue is one of the basic symptoms of FMS. Yilmaz et al. ${ }^{18}$ evaluated muscle fatigue by 30 sequential knee flexionextension motions using isokinetic dynamometer at maximum intensity and at an angular speed of $180 \%$ second. The sum of flexor and extensor works was recorded as total work. In the FMS group, a reduction by 36\% was detected in isokinetic total work and by $32 \%$ in relative total work (taking body weight as the basis). Reduction in muscle endurance was consistent with the reduction in isokinetic muscle strength. ${ }^{18}$ Maquet et al. ${ }^{24}$ detected $40 \%$ decreases in muscle fatigue resistance in FMS, and Akyol et al. ${ }^{19}$ reported greater fatigue scores in the FMS patients. Moreover, they reported more fatigue in FMS patients having more pain severity but no association between fatigue and muscle strength or walking distance. ${ }^{19}$ Valkeinen et al. ${ }^{6}$ conducted a study in elderly FMS patients and elderly healthy females and reported that the FMS patients benefited from strength training as much as healthy subjects and that functional performance of the patients was enhanced. They found increased strength and high-load performance and attenuated perceived pain. We detected no differences between the groups in terms of the fatigue indexes of the wrist flexors and extensors. Our results showed that prolonged high intensity exercises are needed to evaluate muscle fatigue.

In the present study, while there were differences between the groups in terms of weekly hours of walking and house cleaning, no differences were found for weekly hours of bicycling, gardening, doing sports, and total physical activity. Likewise, Kop et al. ${ }^{25}$ reported no difference between the healthy controls and combined patient group (FMS and chronic fatigue syndrome) for total activity. In a study by Korszun et al., ${ }^{26}$ no difference was indicated between the FMS patients and healthy controls regarding total daytime activity. In the abovementioned two studies, the physical activity was measured using an accelerometer. McLoughlin et al. ${ }^{27}$ compared levels of physical activity between the FMS patients and healthy controls using both objective and self-report measures and demonstrated that physically activity levels were lower in the FMS patients. They indicated that physical activity levels were associated with depressed mood rather than being predictive of pain. Thus, they suggested not using only the self-report measures to evaluate physical activity levels because the manner of self-report could quietly vary in FMS patients as compared to healthy controls. In the present study, absence of difference between the groups regarding total physical activity may have resulted from the use of self-report measures alone.

There are a few important limitations in this study. First, our sample size was small and, all of our patients were female. Second, the patients were not questioned about the history of medication usage. Third, although all of the subjects were housewives, their education status was not recorded. Last, total physical activity was questioned in terms of the frequency and duration of daily activities, but an estimate of intensity to 
express metabolic equivalent intensity was not calculated.

In conclusion and in the light of our results, we may indicate that muscle strength tends to decrease in FMS patients' wrist extensor/flexor muscles. We believe that further studies with larger participants are needed to explain the relationship between upper limb muscle performance and FMS, as well as the underlying pathogenesis.

\section{Declaration of conflicting interests}

The authors declared no conflicts of interest with respect to the authorship and/or publication of this article.

\section{Funding}

The authors received no financial support for the research and/or authorship of this article.

\section{REFERENCES}

1. Clauw DJ. Fibromyalgia: a clinical review. JAMA 2014;311:1547-55.

2. Rahman A, Underwood M, Carnes D. Fibromyalgia. BMJ 2014;348:1224.

3. Giacomelli C, Sernissi F, Sarzi-Puttini P, Di Franco M, Atzeni F, Bazzichi L. Fibromyalgia: a critical digest of the recent literature. Clin Exp Rheumatol 2013;31:153-7.

4. Bengtsson A. The muscle in fibromyalgia. Rheumatology (Oxford) 2002;41:721-4.

5. Elvin A, Siösteen AK, Nilsson A, Kosek E. Decreased muscle blood flow in fibromyalgia patients during standardised muscle exercise: a contrast media enhanced colour Doppler study. Eur J Pain 2006;10:137-44.

6. Valkeinen $\mathrm{H}$, Häkkinen A, Hannonen P, Häkkinen $\mathrm{K}$, Alén $\mathrm{M}$. Acute heavy-resistance exerciseinduced pain and neuromuscular fatigue in elderly women with fibromyalgia and in healthy controls: effects of strength training. Arthritis Rheum 2006;54:1334-9.

7. Jacobsen S, Wildschiødtz G, Danneskiold-Samsøe B. Isokinetic and isometric muscle strength combined with transcutaneous electrical muscle stimulation in primary fibromyalgia syndrome. $\mathrm{J}$ Rheumatol 1991;18:1390-3.

8. Lindh MH, Johansson LG, Hedberg M, Grimby GL. Studies on maximal voluntary muscle contraction in patients with fibromyalgia. Arch Phys Med Rehabil 1994;75:1217-22.

9. Nørregaard J, Bülow PM, Vestergaard-Poulsen P, Thomsen C, Danneskiold-Samøe B. Muscle strength, voluntary activation and cross-sectional muscle area in patients with fibromyalgia. $\mathrm{Br} \mathrm{J}$ Rheumatol 1995;34:925-31.

10. Henriksen M, Lund H, Christensen R, Jespersen A, Dreyer L, Bennett RM, et al. Relationships between the fibromyalgia impact questionnaire, tender point count, and muscle strength in female patients with fibromyalgia: a cohort study. Arthritis Rheum 2009;61:732-9.

11. Borman P, Celiker R, Hasçelik Z. Muscle performance in fibromyalgia syndrome. Rheumatol Int 1999;19:27-30.

12. Wolfe F, Smythe HA, Yunus MB, Bennett RM, Bombardier C, Goldenberg DL, et al. The American College of Rheumatology 1990 Criteria for the Classification of Fibromyalgia. Report of the Multicenter Criteria Committee. Arthritis Rheum 1990;33:160-72.

13. Buffart LM, Thong MS, Schep G, Chinapaw MJ, Brug J, van de Poll-Franse LV. Self-reported physical activity: its correlates and relationship with health-related quality of life in a large cohort of colorectal cancer survivors. PLoS One 2012;7:36164.

14. Sarmer S, Ergin S, Yavuzer G. The validity and reliability of the Turkish version of the Fibromyalgia Impact Questionnaire. Rheumatol Int 2000;20:9-12.

15. Forthomme B, Croiser JL, Foidart-Dessalle M, Crielaard JM. Isokinetic assesment of the forearm and wrist muscles. Isokinetic Exerc Sci 2002;10:121-8.

16. Mastaglia FL. The relationship between muscle pain and fatigue. Neuromuscul Disord 2012;22:178-80.

17. Mengshoel AM, Førre O, Komnaes HB. Muscle strength and aerobic capacity in primary fibromyalgia. Clin Exp Rheumatol 1990;8:475-9.

18. Yilmaz H, Ugurlu H, Sallı A. Muscle performance in patients with fibromyalgia syndrome. Rheumatism 2007;22:43-7.

19. Akyol Y, Ulus Y, Tander B, Bilgici A, Kuru O. Muscle strength, fatigue, functional capacity, and proprioceptive acuity in patients with fibromyalgia. Turk J Phys Med Rehab 2013;59:292-8.

20. Yetişgin A, Tiftik T, Kara M, Karabay İ, Akkuş S, Ersöz M. Isokinetic muscle performance of the hip and ankle muscles in women with fibromyalgia. Int J Rheum Dis 2013 Oct 16. [Epub ahead of print]

21. Nordenskiöld UM, Grimby G. Grip force in patients with rheumatoid arthritis and fibromyalgia and in healthy subjects. A study with the Grippit instrument. Scand J Rheumatol 1993;22:14-9.

22. Sahin G, Ulubaş B, Calikoğlu M, Erdoğan C. Handgrip strength, pulmonary function tests, and pulmonary muscle strength in fibromyalgia syndrome: is there any relationship? South Med J 2004;97:25-9.

23. Köklü K, Sarı̈ül M, Özişler Z, Şirzai H, Özel S. Handgrip strength in fibromyalgia. Arch Rheumatol 2016;31:i-iv.

24. Maquet D, Croisier JL, Renard C, Crielaard JM. 
Muscle performance in patients with fibromyalgia. Joint Bone Spine 2002;69:293-9.

25. Kop WJ, Lyden A, Berlin AA, Ambrose K, Olsen $\mathrm{C}$, Gracely $\mathrm{RH}$, et al. Ambulatory monitoring of physical activity and symptoms in fibromyalgia and chronic fatigue syndrome. Arthritis Rheum 2005;52:296-303.

26. Korszun A, Young EA, Engleberg NC, Brucksch
CB, Greden JF, Crofford LA. Use of actigraphy for monitoring sleep and activity levels in patients with fibromyalgia and depression. J Psychosom Res 2002;52:439-43.

27. McLoughlin MJ, Colbert LH, Stegner AJ, Cook DB. Are women with fibromyalgia less physically active than healthy women? Med Sci Sports Exerc 2011;43:905-12. 